\title{
A Validated Preclinical Animal Model for Primary Bone Tumor Research
}

Wagner F. et al.

J Bone Joint Surg Am 2016 Jun 01; 98 (11): 916-925.

This report aimed to describe the engineering and validation of a humanized tissue-engineered bone organ (hTEBO) for preclinical research on primary bone tumors. The harvested pelvic bone and marrow fragments were taken from the patients while reaming the acetabulum during hip arthroplasty. HTEBOs were engineered by embedding fragments in a fibrin matrix containing bone morphogenetic protein-7 and implanted into NOD-SCiD mice. One group of hTEBOs was harvested to analyze the degree of humanization after 10 weeks of subcutaneous growth. The second group was injected with human luciferase-labeled OS (LucSAOS-2) cells, and tumor growth was followed in vivo with bioluminescence imaging. The OS tumors were harvested and analyzed after 5 weeks and compared with the tumors created via intratibial injection.

A new bone organ containing human bone matrix as well as viable and functional human hematopoietic cells developed after 10 weeks of in vivo growth. Five weeks after injecting Luc-SAOS-2 cells into this humanized bone microenvironment, spontaneous metastatic spread to the lung was evident.

The study reported an in vivo model that contained human bone matrix and marrow components in one organ. This novel platform might enable preclinical research on primary bone tumors to test new treatment options.

\section{Effectiveness of Aquatic Exercises in Women with Rheumatoid Arthritis: A Randomized, Controlled, 16-Week Intervention - The HydRA Trial}

Siqueira U.S. et al.

Am J Phys Med Rehabil 2016 Jul 6.

This study aimed to evaluate the effectiveness of two different types of aerobic exercises [land-based (LB) and water-based (WB)] in women with rheumatoid arthritis (RA).

A total of 133 women with RA were included in this prospective, randomized, blinded, 16-week controlled study. The patients were separated into three groups as WB $(n=33), L B(n=33)$, and control $(n=34)$. An isokinetic dynamometer was used to evaluate the muscle strength of knees. The same expert rheumatologist evaluated disease activity (DAS-28) and functional status (health assessment questionnaire). Total body densitometry was used to measure body composition. The treatment was applied three times per week, and all groups were assessed at baseline and after 8 and 16 weeks. Additionally, the compliance of patients and concomitant medications was evaluated. A P value less than 0.05 was considered significant.

Eighty-two patients completed the study. Before treatment, the three groups were matched for age, body composition, functional status, muscle strength, and concomitant medications. At the end of the treatment, no significant changes in knee muscle strength and body composition were found among the three groups. However, a significant improvement in disease activity and functional capacity was observed in the WB group after 8 and 16 weeks.

Aquatic aerobic exercises seem to be more effective in women with RA.

* This update summarizes selected articles that were published in 2016 in distinguished medical scientific journals.

** Prepared by:

Şinasi ÖZSOYLU, MD e-mail: sinasiozsoylu@hotmail.com F. Gülçin UĞURLU, MD e-mail: glcnrl@hotmail.com 


\section{Viremia and Ebola Virus Secretion in Survivors of Ebola Virus Disease in Sierra Leone: A Cross-sectional Cohort Study}

Green E. et al.

Lancet Infect Dis 2016; 16: 1052-56

The 2014 Ebola outbreak in West Africa was the largest Ebola outbreak in history. This cross-sectional cohort study aimed to evaluate the risk of infection of survivors to health care providers. Axillary, blood, conjunctival, forehead, mouth, rectal, semen, urine, and vaginal specimens were collected from the survivors of Ebola virus disease in Sierra Leone. The existence of Ebola virus was investigated in these samples by reverse transcription-polymerase chain reaction (RT-PCR).

Patients who were treated at the Kerry Town Ebola Treatment Unit (ETU) between November 5, 2014, and March 24, 2015, were re-evaluated at the Kerry Town survivor clinic. Whether the survivors had acute Ebola virus infection was assessed in this clinic. Patients who met the case definition criteria for acute Ebola virus disease or who had positive Ebola virus RT-PCR on whole blood were excluded from the study.

Between April 2, and June 16, 2015, 555 samples were collected from 112 survivors. The median time between ETU discharge and sample collection was 142 days (interquartile range: 127-159). Only one semen sample was collected and found to be positive for Ebola virus. This sample was collected on the 114th day of the discharge of a patient from the ETU. All other samples collected from 111 survivors were found to be negative for Ebola virus RT-PCR.

It was concluded that the survivors of Ebola virus disease who were not compatible with the case definition for acute Ebola disease 6 weeks after the clearance of viremia probably had low infectivity. Therefore, standard barrier precautions might be sufficient for health care workers after this time if no probable contact with fluids from sanctuary sites exists. 